\title{
Response Characteristics of an Identified, Sexually Dimorphic Olfactory Glomerulus
}

\author{
Jane Roche King, Thomas A. Christensen, and John G. Hildebrand \\ Arizona Research Laboratories, Division of Neurobiology, University of Arizona, Tucson, Arizona 85721-0077
}

Partitioning of synaptic neuropil into glomeruli is a common feature of primary olfactory centers in most animal species. The functional significance of glomeruli, however, is not yet well understood. The present study is part of our effort to test the hypothesis that each glomerulus is a functional unit dedicated to processing information about a particular odorant or attribute of odor molecules and that the glomerular array constitutes a map of "odor space." We investigated the physiological and morphological features of uniglomerular projection neurons (PNs) associated with an identified glomerulus in each antennal lobe of the female sphinx moth, Manduca sexta. This "lateral large female glomerulus" (latLFG) is sexually dimorphic and therefore may play a female-specific role, such as processing of information about one or more odorants important for orientation of a female to host plants for oviposition. Together with the medial LFG (medLFG), the latLFG resides outside the array of spheroidal ordinary glomeruli, near the entrance of the antennal (olfactory) nerve. Each LFG is innervated by four to five PNs. Using intracellular recording and staining, we examined the responses of latLFG-PNs to odorants that represent major classes of volatiles released by host plants of $M$. sexta. All latLFG-PNs were excited when the ipsilateral antenna was stimulated with low concentrations of the monoterpenoid linalool. Dose-response analysis showed that neither other monoterpenoids nor representatives of other classes of host plant volatiles were similarly stimulatory to latLFG-PNs. These findings are consistent with the idea that each glomerulus has a characteristic, limited molecular receptive range.

Key words: olfaction; antennal lobe; glomeruli; sexual dimorphism of the CNS; insect; Manduca sexta
Unlike the visual, auditory, and somatosensory systems, the olfactory system lacks a precise spatial map of the periphery. How central neural space is used to map olfactory information is only beginning to be understood. Primary olfactory centers typically exhibit condensed neuropil structures called glomeruli. According to a long-established hypothesis, each glomerulus is a functional unit dedicated to processing information about a particular odorant or attribute of odor molecules, and the glomerular array constitutes a chemotopic map of "odor space" (for review, see Hildebrand, 1995; Buck, 1996; Hildebrand and Shepherd, 1997; Christensen and White, 2000).

Activity-dependent labeling with 2-deoxyglucose or voltage- or $\mathrm{Ca}^{2+}$-sensitive dyes has revealed that odors elicit reproducible patterns of activity across the glomerular array in the vertebrate olfactory bulb and insect antennal lobe (AL) (Teicher et al., 1980; Rodrigues and Buchner, 1984; Rodrigues, 1988; Kauer and Cinelli, 1993; Friedrich and Korsching, 1997, 1998; Joerges et al., 1997; Distler et al., 1998). In addition, single-unit recordings have shown that neighboring output neurons, presumably with arborizations in the same glomerulus, are more likely to respond similarly to individual odorants than are more widely separated output neurons associated with different glomeruli (Buonviso and Chaput, 1990; Chaput, 1990). Such studies have supported the

Received Oct. 21, 1999; revised Dec. 23, 1999; accepted Dec. 28, 1999.

This work was supported by National Institutes of Health Grant DC02751. We thank Heather Stein, Patricia Jansma, Nirav Merchant, and Amit Pandy for expert technical assistance; John Douglass for writing the spike-analysis software; A. A. Osman and Zenzele Mpofu for rearing Manduca; and Reg Chapman, Ann Fraser, and Eileen Hebets for helpful comments on this manuscript.

Correspondence should be addressed to Dr. John G. Hildebrand, Arizona Research Laboratories, Division of Neurobiology, University of Arizona, 611 GouldSimpson Building, 1040 E. 4th Street, Tucson, AZ 85721-0077. E-mail: jgh@ neurobio.arizona.edu.

Copyright (C) 2000 Society for Neuroscience $\quad 0270-6474 / 00 / 202391-09 \$ 15.00 / 0$ idea that glomeruli are functionally significant modules but have not tested their specificity by examining the anatomical and physiological properties of output neurons from identified glomeruli.

To understand glomerular function, it is important to study glomeruli that are identifiable across individuals, a prerequisite that has been met by several insect systems (Chambille et al., 1980; Vickers et al., 1998; Rospars and Hildebrand, 1992, 2000). In the sphinx moth, Manduca sexta, for example, the male-specific macroglomerular complex (MGC) is a cluster of identified glomeruli dedicated to processing information about individual components of the conspecific female's sex pheromone (Hildebrand, 1996). Little is known, however, about the olfactory tuning of other glomeruli in the ALs.

Female $M$. sexta use olfactory information to locate host plants for oviposition (Willis and Arbas, 1991). Recently, a pair of sexually dimorphic glomeruli has been described in the female AL (Rössler et al., 1998; Rospars and Hildebrand, 2000). These LFGs are particularly attractive for studies of glomerular function because they are identified, characteristically situated, and therefore favorable for physiological recording.

We have begun to examine the functions of the LFGs and to test the hypothesis of chemotopy by studying anatomical features and molecular receptive ranges (Mori and Shepherd, 1994) of output neurons with arborizations confined to the LFGs. For olfactory stimulation, we used single compounds prominent in headspace-volatile mixtures emitted by host plants. Here we report that the lateral LFG responds best to only one of a selected set of odorants representing three chemical classes and thus may have a narrow molecular receptive range. Our findings suggest that like the glomeruli of the chemotopically organized male's 
MGC, the LFGs process olfactory information about a limited range of odor chemistry.

A preliminary report of some of this work has appeared previously (King et al., 1998).

\section{MATERIALS AND METHODS}

Preparation. Manduca sexta (L.) (Lepidoptera: Sphingidae) were reared in the laboratory on an artificial diet under a long-day photoperiod regimen, as described previously (Sanes and Hildebrand, 1976). Adult female moths were used 1-4 d after emergence and prepared for experiments as described previously (Christensen and Hildebrand, 1987). For electrophysiological recording, a moth was restrained in a plastic tube with its head exposed at one end. The labial palps, proboscis, and cibarial musculature were then removed to allow access to brain. To eliminate movement, the head of the moth was isolated and pinned to a Sylgardcoated glass Petri dish with the ALs facing upward. Tracheae and a small part of the sheath overlying one AL were removed with fine forceps. The preparation was continuously superfused for the duration of the experiment with physiological saline solution containing (in $\mathrm{mM}$ ): $150 \mathrm{NaCl}, 3$ $\mathrm{CaCl}_{2}, 3 \mathrm{KCl}, 10$ TES buffer, and 25 sucrose, $\mathrm{pH} 6.9$.

Stimulation. After the head of the moth had been pinned to the Sylgard-coated dish, the tip of one antenna was removed, and the cut end of the antenna was inserted into a thin-walled borosilicate capillary tube filled with physiological saline solution. This capillary served both as a holder to suspend the antenna and as an electrode for electroantennogram recording to monitor responses of the antenna to stimuli. Once the antenna was suspended, a glass tube carrying a stream of humidified, charcoal-filtered air was positioned $\sim 10 \mathrm{~mm}$ in front of the antenna. Odorants were injected into this continuous air stream by means of a motor-driven syringe olfactometer (Selchow, 1998). The olfactometer was activated by a computer-controlled command pulse using customized ASYST software (Keithly Instruments, Rochester, NY). For most trials, a single, $200 \mathrm{msec}$ odor pulse was delivered to the antenna, but in some cases, five sequential $50 \mathrm{msec}$ pulses were presented.

Because an intracellular impalement could be held for no more than $40-50 \mathrm{~min}$ at best, there was sufficient time to test several concentrations of only a selected few odor stimuli in this effort to characterize the neurons of the latLFG. The odorants used for characterization of neuronal response profiles represent three chemical classes: the monoterpenoids linalool ([ \pm$] 3,7$-dimethyl-1,6-octadien-3-ol, catalog no. L-5255; Sigma, St. Louis, MO), nerol (cis-3,7-dimethyl-2,6-octadien-1-ol, catalog no. 26,890-9; Aldrich, Milwaukee, WI), $\beta$-ocimene (3,7-dimethyl-1,3,6octatriene, catalog no. 74730; Fluka, Buchs, Switzerland), and $\beta$-myrcene (3-methylene-7-methylocta-1,6-octadiene, catalog no. M 0382; Sigma); an aliphatic aldehyde, trans-2-hexenal [(t-2-h) catalog no. H 6765, Sigma]; and an aromatic ester, methyl salicylate [(ms) methyl-2-hydroxybenzoate, catalog no. M 6752, Sigma]. These compounds (Fig. 1) are produced by the vegetative and/or floral parts of host plants of M. sexta (Buttery et al., 1987; Andersen et al., 1988; Loughrin et al., 1990; Knudsen et al., 1993; Raguso and Willis, 1997; W. Mechaber and J. G. Hildebrand, unpublished observations). In addition to these single compounds, the blend of headspace volatiles released in a syringe by a single fresh leaf from a tomato plant (a preferred host plant of $M$. sexta) was used as a stimulus. Single odor compounds were diluted to various concentrations in odorless light mineral oil (Sigma). A total of $50 \mu \mathrm{l}$ of the final dilution was applied to a $20 \mathrm{~mm}$ disk of Whatman number-1 filter paper. The odorbearing filter paper was immediately inserted into a capped $20 \mathrm{ml}$ syringe, which was allowed to equilibrate for at least $4 \mathrm{hr}$ before use to ensure quantitatively reproducible odor delivery (Selchow, 1998). Dilutions resulted in a final application of $0.005 \mu \mathrm{l}\left(1: 10^{4}\right)$ to $5 \mu \mathrm{l}(1: 10)$ of pure compound on the filter paper. Based on differences in the specific gravities and purities of the compounds, the final gravimetric loads at the $1: 10^{3}$ dilution ranged from $35.56 \mu \mathrm{g}$ for $\beta$-myrcene to $58.41 \mu \mathrm{g}$ for methyl salicylate per $50 \mu \mathrm{l}(\sim 1 \mu \mathrm{g} / \mu \mathrm{l}$ for all compounds).

Intracellular recording and staining. Intracellular recording was performed as described previously (Christensen and Hildebrand, 1988). Borosilicate glass microelectrodes filled with a $4 \%$ solution of Lucifer Yellow CH (LY) (Sigma) in $0.2 \mathrm{M} \mathrm{LiCl}$, and having resistances of 100-400 M $\Omega$, were used for intracellular recording and staining. Microelectrodes were manipulated to penetrate the glomerular region of the AL above the known location of the LFGs, so that LFG-PNs could be targeted in these experiments. The responses of PNs to stimulation of the ipsilateral antenna with odorants were monitored on an oscilloscope and directly digitized with a computer using Axoscope software (Version 1.1,

\section{Host-Plant Volatiles}

\section{Monoterpenoids}
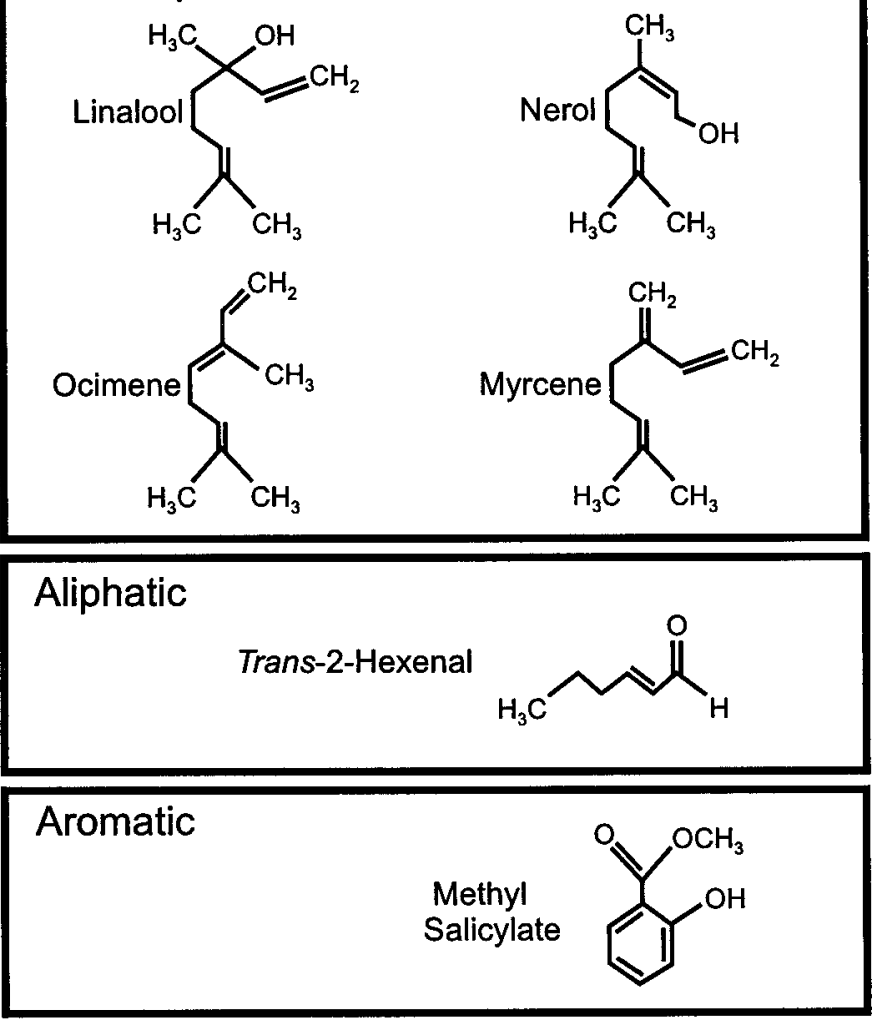

Figure 1. Chemical structure of host plant-related volatiles used to test the responses of uniglomerular PNs associated with the latLFG. Three classes of odorants were used: monoterpenoids (top panel), an aliphatic aldehyde (middle panel), and an aromatic ester (bottom panel). Note the structural relatedness of the monoterpenoids.

Axon Instruments, Foster City, CA). After physiological characterization, cells were injected with LY by passing hyperpolarizing current (up to $1 \mathrm{nA}$ ) for 5-15 min. Longer injections usually resulted in more dense and complete staining of neurons.

Immediately after the completion of an experiment, the brain was excised and immersed in formaldehyde fixative solution (2.5\% formaldehyde in $0.1 \mathrm{M}$ phosphate buffer with $3 \%$ sucrose added) in a glass vial. Brains were fixed for at least $1 \mathrm{hr}$, after which they were dehydrated by passage through a graded series of ethanol solutions and finally cleared with methyl salicylate. Brains used for initial glomerular identification were post-fixed in $2 \%$ glutaraldehyde in $0.1 \mathrm{M}$ phosphate buffer to enhance contrast. Cleared brains were viewed as whole mounts with a laser-scanning confocal microscope [Bio-Rad MRC-600 (Bio-Rad, Cambridge, MA) equipped with a Nikon Optiphot-2 microscope and both 15 $\mathrm{mW}$ krypton/argon and $100 \mathrm{~mW}$ argon laser light sources]. Preparations found to contain stained neurons were returned to $100 \%$ ethanol and embedded in Spurr's resin (Electron Microscopy Sciences, Ft. Washington, PA) in preparation for serial sectioning. Sections $(48 \mu \mathrm{m})$ were then cut on a sliding microtome, and images were collected from each section at $2 \mu \mathrm{m}$ optical steps with the confocal microscope. The stained neuron was reconstructed from these images so that the glomerulus housing its arborization could be determined.

Data analysis. Frequency analysis of spike trains was performed with the aid of a DOS-based program written in Turbo Pascal by Dr. J. Douglass (ARL Division of Neurobiology, University of Arizona). Electrophysiological records saved as text files were imported into the program, where they were converted to instantaneous spike frequency (ISF) values. These values were then plotted against time of occurrence of each spike to yield histograms plotting instantaneous frequency versus time. For dose-response curves, the peak value of ISF obtained during each 6 sec recording period was used to calculate the mean ( \pm SEM) for each odor compound at each concentration. 
Three-dimensional reconstructions. Methods described by Vickers et al. (1998) were used for three-dimensional (3-D) reconstruction of ALs containing LY-injected neurons. Briefly, $2 \mu \mathrm{m}$ optical sections from glutaraldehyde-fixed whole mounts and from $48 \mu \mathrm{m}$ Spurr's sections were collected with the laser-scanning confocal microscope. Using Confocal Assistant software (version 4.02, Todd Brelje, Bio-Rad), five individual optical sections were stacked to produce an image representing an optical slab of tissue $\sim 10 \mu \mathrm{m}$ thick. These images were then printed with a 600 dpi laser printer. The borders of the AL and individual glomeruli were traced and digitized using a digitizing tablet (model XGT, Kurta, Phoenix, AZ) to yield a bitmap file representing each section. These bitmap files were then rendered in 3-D, using software custom-designed by Nirav Merchant and Amit Pandy (ARL Division of Neurobiology, University of Arizona), on a Silicon Graphics (Mountain View, CA) Indigo computer. With Cosmo Worlds software (Silicon Graphics), 3-D images were rotated in any plane to allow visualization of glomeruli from various aspects.

\section{RESULTS}

\section{Spatial organization of the large female glomeruli}

With the aid of 3-D reconstructions, we have identified morphologically a number of glomeruli in the AL of $M$. sexta that are favorable, by virtue of their size and location, for physiological studies. Among these are two prominent glomeruli in the female AL, previously designated "lateral female glomeruli" (Rössler et al., 1998). In view of their locations within the AL and their sizes, these two identified, sexually dimorphic glomeruli have been renamed the large female glomeruli (Rospars and Hildebrand, 2000). Based on 3-D reconstructions of eight ALs from eight different female moths, we have found that like the MGC in each AL of male moths (Matsumoto and Hildebrand, 1981; Hansson et al., 1991; Vickers et al., 1998), the LFGs reside near the entrance of the antennal nerve into the AL, outside the array of ordinary glomeruli (Fig. $2 A, B$ ). The LFGs directly abut each other, with one, designated the latLFG, at the lateral edge of the AL. This glomerulus lies slightly anterior to the more posteromedially situated LFG, designated the medLFG (Fig. $2 C$ ). The sizes of the LFGs and their positions, relative to landmarks such as the entrance of the antennal nerve and the lateral group of neuronal cell bodies in each AL, are relatively constant among individuals, so that the LFGs are readily identifiable in confocal stacks. Each LFG is innervated by the axon terminals of numerous olfactory receptor cells (ORCs), at least four to five uniglomerular projection (output) neurons (PNs) (Fig. 3), a number of multiglomerular local interneurons, and possibly multiglomerular projection neurons (Homberg et al., 1988). In this study, we examined olfactory responses of the uniglomerular PNs.

\section{Physiological response properties of uniglomerular projection neurons innervating the lateral large female glomerulus}

Because glomerular assignment was crucial to this study of AL PNs, analysis and conclusions are based only on neurons that were physiologically characterized and stained intracellularly with Lucifer Yellow. We report on 10 physiologically characterized and stained PNs, in 10 different female moths, which had dendritic arborizations restricted to the latLFG (Figs. 3A, 4). Each of these PNs had its soma located in the medial group of AL neuronal somata (Homberg et al., 1988). In five preparations in which the PN axons were completely stained, it could be ascertained that these PNs were PIa neurons, based on the nomenclature of Homberg et al. (1988). The axons of these PNs projected via the inner antenno-cerebral tract to the calyces of the ipsilateral mushroom body and the lateral horn of the protocerebrum (Fig. 4E,F,G).
A
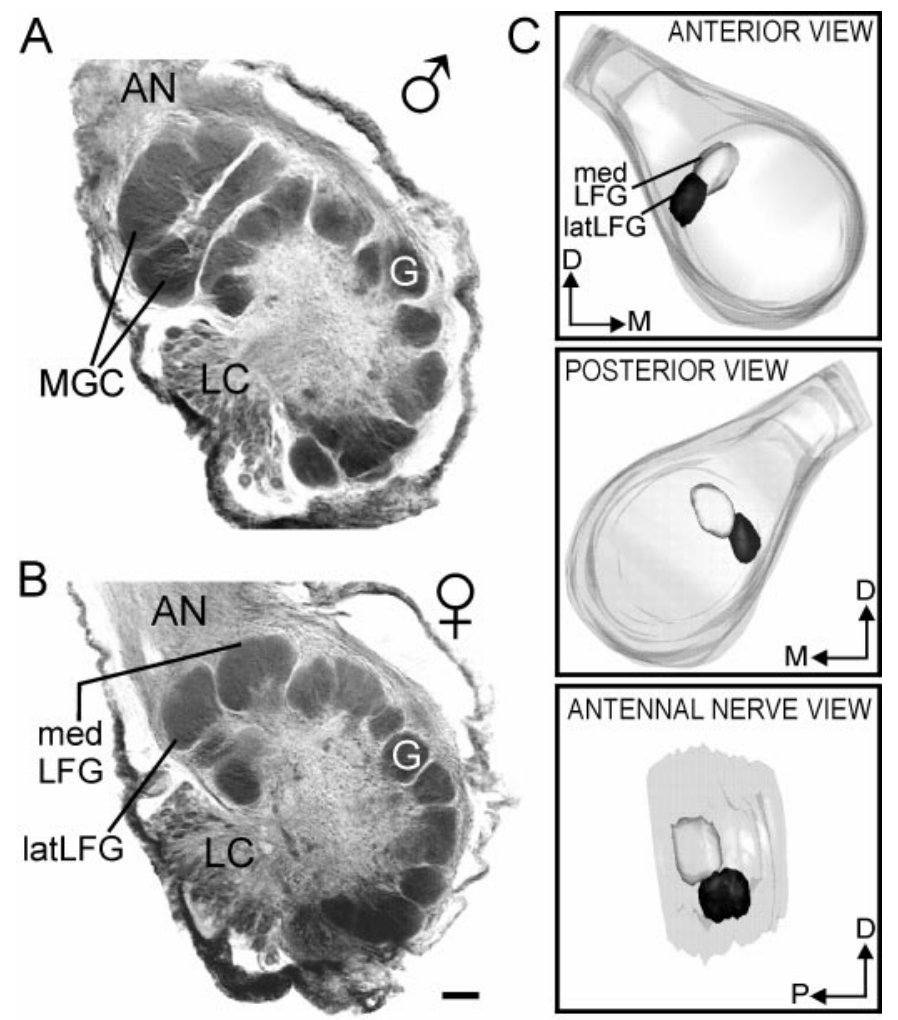

Figure 2. Sexually dimorphic glomeruli in M. sexta. $A, B$, Confocal images of ALs from a male $(A)$ and a female $(B) M$. sexta. In the male $\mathrm{AL}$, the macroglomerular complex $(M G C)$ resides at the entrance of the antennal nerve $(A N)$, outside of the shell-like array of spheroidal "ordinary" glomeruli $(G)$. Occupying a similar position in the female AL are the two LFGs. The lateral group of neuronal cell bodies $(L C)$ appears in each section. $C, 3-\mathrm{D}$ reconstruction of a female AL showing the positions of the medLFG and latLFG from three different views. The top panel shows a frontal view, the middle panel shows a back or posterior view, and the bottom panel shows a view of the LFGs along the axis of the antennal nerve. The medLFG is shown in light gray, and the latLFG is shown in black. $D$, Dorsal; $M$, medial; $P$, posterior. Scale bar, $50 \mu \mathrm{m}$.

Intracellular recordings from latLFG-PNs showed that linalool was strongly excitatory even at the lowest concentration tested (Figs. 5, 6). Increasing the concentration of linalool led to increases in ISF during the initial excitatory phase of the responses (Fig. 5, insets) (at 1:10,000, peak ISF was $140.6 \mathrm{sec}^{-1}$; at 1:1000, peak ISF was $\left.196.08 \mathrm{sec}^{-1}\right)$. A higher concentration of linalool also resulted in a decrease in the latency of the excitatory response (Fig. 5) (at 1:10,000, latency from the beginning of the stimulus pulse to the first spike was $302.3 \mathrm{msec}$; at 1:1000, latency was $220.6 \mathrm{msec}$ ). The excitatory phase of the response to linalool was followed by membrane hyperpolarization, resulting in cessation of spiking activity (Figs. 5, 6). The duration of both the excitatory and inhibitory phases increased with increasing concentration. A summary of these physiological findings is presented in Table 1.

The two other single odorants ( $\mathrm{t}-2-\mathrm{h}$ and $\mathrm{ms}$, even at 10 -fold higher concentration), the blend of tomato-leaf volatiles, and the mineral oil blank all failed to evoke an excitatory response from these PNs (Figs. 5, 6). Higher concentrations of the single odorants sometimes elicited an inhibitory response resulting in a cessation of background spiking activity (Fig. 5).

These observations were consistent across all 10 PNs that had arborizations exclusively in the latLFG (Fig. 7). Each latLFG-PN 

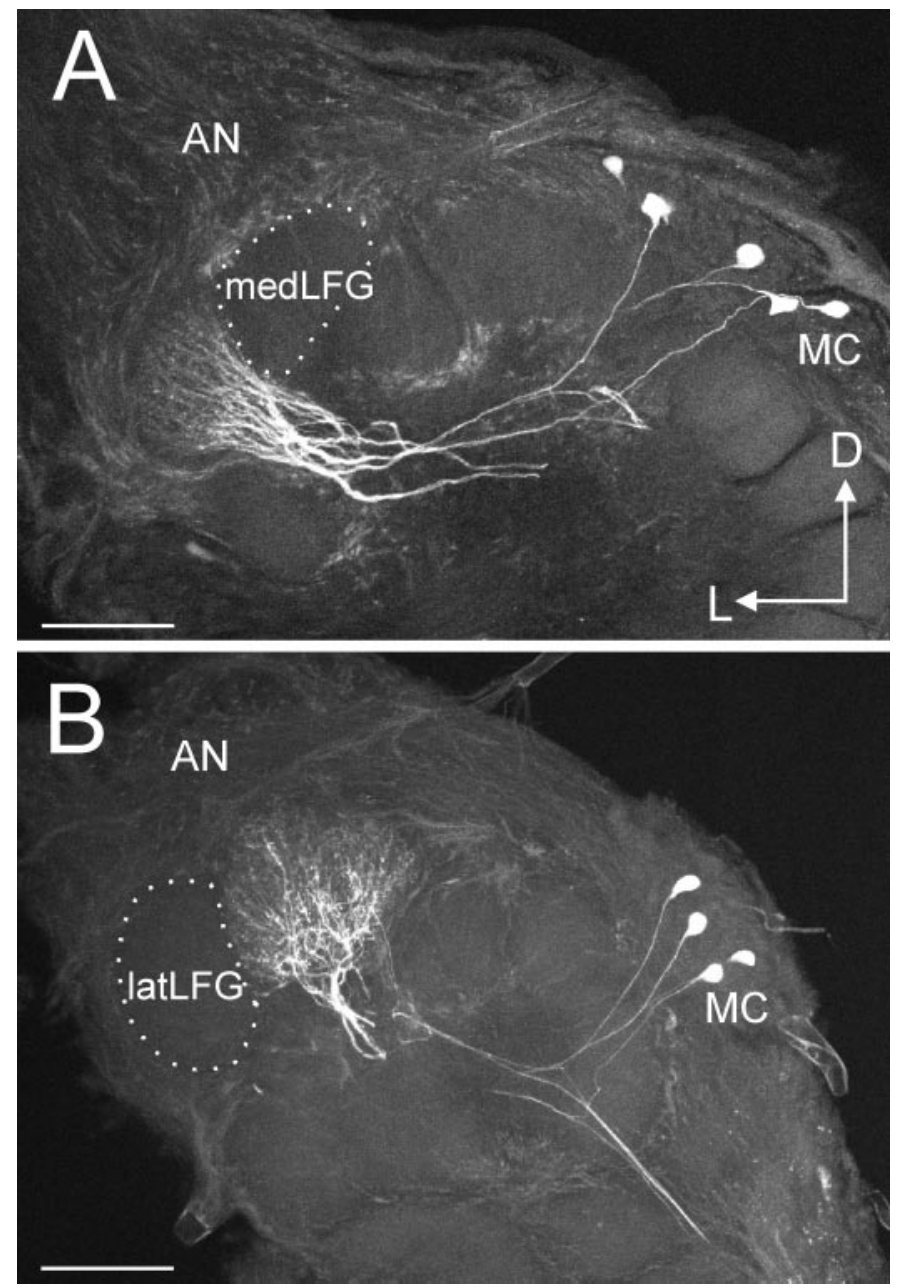

Figure 3. Multiple uniglomerular projection neurons with arborizations confined to an LFG. A, A confocal stack showing four or five PNs with dendritic arborizations restricted to the latLFG. The medLFG, directly adjacent to the latLFG, contains no neurites of these PNs. $B$, A confocal stack showing four PNs with dendritic arborizations restricted to the medLFG. In this case, the latLFG contains no processes of these neurons. Each of these PNs has a soma located in the medial group of neuronal cell bodies $(M C) . A N$, Antennal nerve; $D$, dorsal; $L$, lateral. Scale bars, $100 \mu \mathrm{m}$.

was strongly and selectively excited in response to antennal stimulation with linalool. Although their response profiles were similar, these PNs varied in their overall sensitivity to linalool as indicated by the variation in the ISF on stimulation with a concentration of 1:1000 (Fig. 7, top row). In addition, two of the PNs (1 and 3) were excited in response to the blend of tomato-leaf volatiles. That stimulus was likely to be variable from one experiment to another, owing to differences among leaf samples used in the stimulus syringe, which probably accounts for the excitation observed in these PNs. It is also possible that these two neurons may represent a different class of latLFG-PNs that respond to other compounds in addition to linalool.

Dose-response analysis further demonstrates the sensitivity and selectivity of latLFG-PNs to linalool (Fig. 8). Some of the latLFG-PNs were tested with a larger set of single odorants at various concentrations to determine whether latLFG-PNs were responsive only to linalool or if other compounds with similar chemical structures could also elicit responses. Data from two latLFG-PNs (Fig. 8) show that linalool was the strongest stimulus, driving the cells to a greater ISF than any of the other odorants delivered at a similar concentration. Of the monoterpenoids tested, only nerol could elicit excitatory responses in these PNs, but with a potency roughly $1 \%$ that of linalool (Fig. 8). The ISF values recorded after delivery of the other compounds, including tomato-leaf volatiles, were comparable to background firing and to the responses to stimulation with the mineral oil blank. Thus, it appears that the latLFG-PNs were narrowly tuned to linalool or a compound related to it. These dose-response curves also suggest that latLFG-PNs may vary in sensitivity to linalool as reflected in the peak ISF for linalool at the various concentrations tested.

In addition to chemical information, the moth's olfactory system also encodes information about the temporal features of odor stimuli (Christensen et al., 1996). Several latLFG-PNs were tested with $50 \mathrm{msec}$ pulses of odor delivered at various rates to test the ability of these PNs to follow pulses of linalool. The latLFG-PNs could follow odor pulses delivered at rates of three or four pulses per second (Fig. 9). Each odor pulse evoked a discrete onset of action potentials that was abruptly terminated by a strong membrane hyperpolarization. This inhibitory mechanism therefore ensures that the neurons will remain inactive during the interval before the next stimulus arrives, thus increasing the "contrast" between odor pulses. This inhibition may last for several seconds, as is evident in the hyperpolarizations evoked by the fifth pulse in the stimulus train of Figure 9. These findings illustrate that the ability of the olfactory system to encode information about the spatiotemporal properties of the stimulus is not limited to males or to pheromone information processing (Hansson and Christensen, 1999).

\section{DISCUSSION}

The insect AL is an experimentally favorable model system for studies of the function of glomeruli in olfactory information processing because (1) the glomerular array is relatively invariant across individuals of a species, and all glomeruli can be identified from individual to individual within a species (Chambille et al., 1980; Rospars, 1983; Rospars and Hildebrand, 1992, 2000); (2) output PNs with arborizations confined to a specific glomerulus are tractable for intracellular and extracellular recording; and (3) many of the odorants important for various aspects of behavior are known.

The lateral and medial LFGs are prominent glomeruli in the ALs of female M. sexta. Although the MGC glomeruli in ALs of male $M$. sexta have been studied extensively, this is the first investigation of the anatomical and physiological properties of neurons with arborizations in the LFGs. Because they are sexually dimorphic and assume their characteristic sizes, shapes, and positions only in the female ALs, the LFGs may play important roles in female-specific behavior, such as upwind orientation toward, and recognition of, host plants and/or oviposition-related cues.

The MGC and LFGs are similarly but distinctively situated in the ALs of males and females, respectively (Fig. 2), and are among the first glomeruli to arise during postembryonic development (Rössler et al., 1998). Recent findings suggest that the two LFGs are homologous to the two main glomeruli of the MGC (Rospars and Hildebrand, 2000). Developmental studies of gynandromorphic female moths with trans-sexually grafted male antennae suggest that PNs that normally arborize within the LFGs are recruited to innervate the induced MGC (Rössler et al., 1999). In addition, some gynandromorphic females with male antennae and induced MGCs orient and fly upwind toward a 

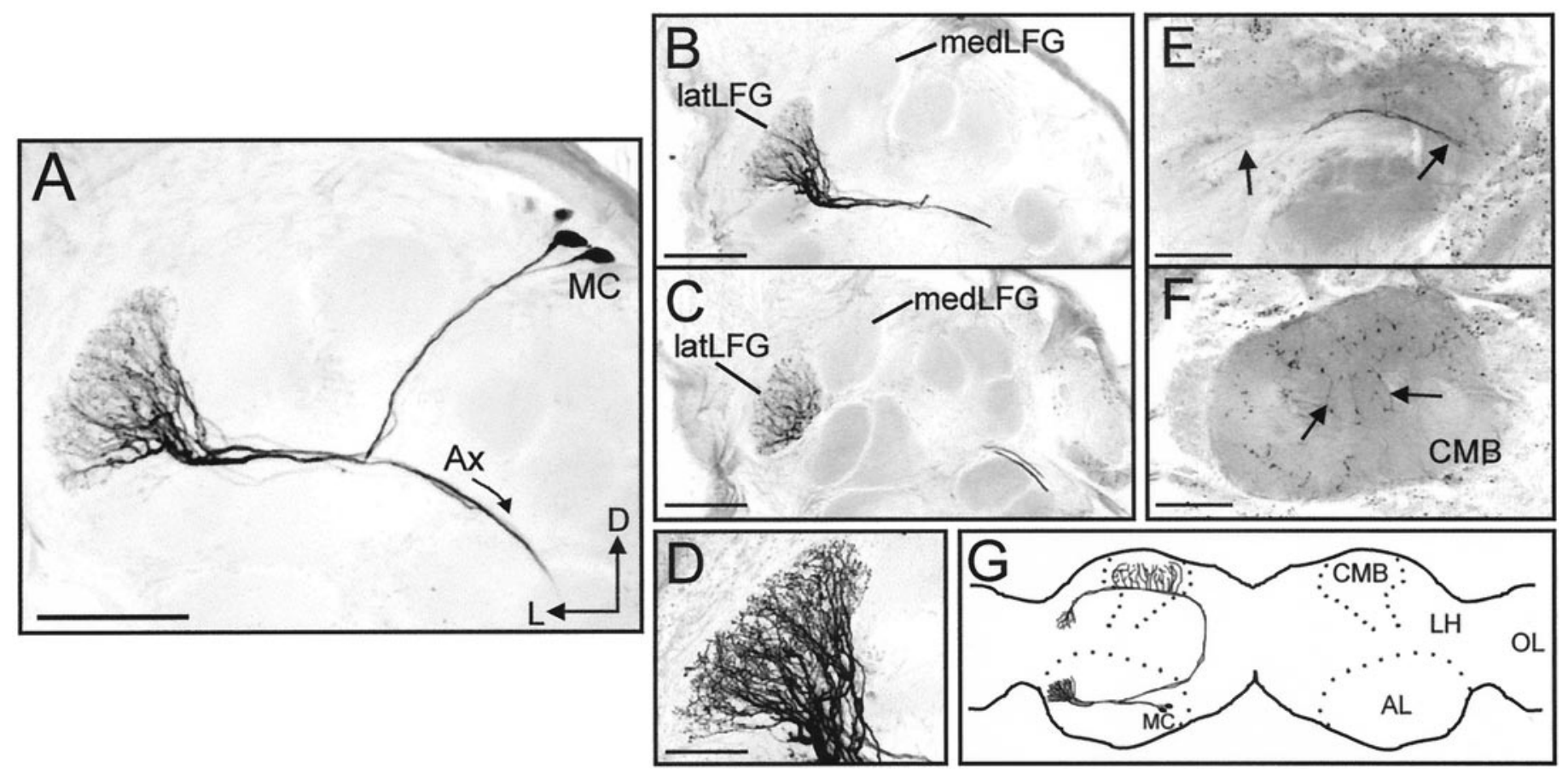

Figure 4. Morphology of uniglomerular PNs arborizing within the latLFG. A, Confocal stack collected from a whole-mount preparation, showing PNs with somata in the medial group of neuronal cell bodies $(M C)$, dendritic arborizations restricted to the latLFG, and axons $(A x)$ projecting from the antennal lobe $(A L) . B, C$, Confocal images collected from the same preparation shown in $A$ after sectioning, illustrating more clearly the close anatomical relationship between the medLFG and latLFG. The dendritic arborizations of these PNs were restricted to the latLFG. Somata are not visible in either section. $D$, High magnification $(60 \times)$ confocal stack of the latLFG shown in $B$. The fine dendritic arborizations of these PNs were highly branched and extended throughout the entire glomerulus. $E, F$, Axonal projection patterns of these latLFG-PNs. Axons (arrows in $E$ ) projected through the inner antennocerebral tract to the calyces of the ipsilateral mushroom body $(C M B)$ and the lateral horn of the protocerebrum. Axon terminals are visible in the CMB indicated in $F$ by arrows. $G$, Schematic illustration (horizontal view) showing the general morphology of latLFG-PNs. All latLFG-PNs observed to date shared the same basic morphology and are classified as type PIa neurons (Homberg et al., 1988). $L H$, Lateral horn of the protocerebrum; $O L$, optic lobe; $D$, dorsal; $L$, lateral. Dotted lines indicate the outlines of the ALs and the mushroom bodies. Scale bars: $A-C, E, F, 100 \mu \mathrm{m} ; D, 50 \mu \mathrm{m}$. All images were inverted using Corel Photo-Paint.

source of sex pheromone, a stimulus to which normal females are unresponsive. On reaching the pheromone source, gynandromorphic females sometimes exhibit oviposition behavior, which is ordinarily elicited by host plant stimuli (Schneiderman et al., 1986). These findings indicate that the MGC and LFGs share common features of development and possibly downstream connections mediating odor-modulated behavior, and also highlight the importance of understanding the functional significance of these identified glomeruli. In moths, glomeruli specific to the female ALs have been described in a few different species (Rospars, 1983; Schneider and Wunderer, 1990), but the roles these glomeruli play in processing of olfactory information or in female behavior are not known.

Female $M$. sexta use olfactory cues to orient toward and locate host plants (Willis and Arbas, 1991; W. Mechaber and J. G. Hildebrand, unpublished observations). By testing latLFG-PNs for their responses to antennal stimulation with various host plant-related volatiles, we have found that the latLFG-PNs respond preferentially to a monoterpenoid, linalool. Although technical constraints limited this study to only six individual odorants, the fact that monoterpenoids similar in structure to linalool did not elicit a comparable excitatory response (Figs. 1,8) suggests that the molecular receptive range of the latLFG may be limited to linalool and perhaps other closely related compounds. The responses to linalool recorded from latLFG-PNs were typically biphasic, with an early excitatory phase followed by a period of hyperpolarization. The peak instantaneous firing frequency of the excitatory phase of the response increased, and the latency to the excitation decreased, with greater odorant concentrations (Table 1). The latLFG-PNs were also capable of following 50 msec pulses of linalool presented at three to four pulses per second. Taken together, these findings indicate that the pattern of spiking in a particular PN depended on both the concentration and the pattern of the odor stimulus (Fig. 9; Table 1). Our observations therefore suggest that latLFG-PNs respond to a particular host plant odorant and encode information about both its concentration and intermittency, as has been documented for MGC-PNs processing sex pheromonal information in the ALs of male moths (Christensen et al., 1998).

Although physiological characteristics of the linaloolresponsive latLFG-PNs described here are similar to those described for sex pheromone-specific MGC-PNs in males (Christensen and Hildebrand, 1987, 1997; Hansson et al., 1991), latLFG-PNs typically were not as sensitive to linalool as MGCPNs are to sex pheromone components. MGC-PNs exhibit response thresholds in the range 0.1-10 ng (Matsumoto and Hildebrand 1981; Hansson et al., 1991), whereas most of the latLFGPNs in this study responded with excitation at a dosage as low as $4.17 \mu \mathrm{g}$ of linalool (Figs. 5, 8, top panel; Table 1).

Whether linalool is behaviorally significant for $M$. sexta is not known. Antennal ORCs that respond preferentially to linalool have been described in female M. sexta (Shields and Hildebrand, 1998), as in the silkmoth, Bombyx mori (Heinbockel and Kaissling, 1996). Linalool is commonly emitted by floral and vegetative parts 
Figure 5. Physiological records collected from one of the stained latLFG-PNs of Figure 4. This neuron was tested with various concentrations of three single odorants [linalool, trans-2-hexenal $(t-2-h)$, methyl salicylate $(\mathrm{ms})$ ], along with the volatile blend emitted by a whole tomato leaf and a mineral oil blank. Stimulus onset is indicated by the vertical dotted line. Stimulus duration was $200 \mathrm{msec}$, marked by the solid line under each record. This neuron was strongly excited when the ipsilateral antenna was stimulated with linalool even at the lowest concentration tested $(1: 10,000)$. Insets in the first two records show a $300 \mathrm{msec}$ segment on an expanded time scale. These insets illustrate the increase in instantaneous spike frequency with an increase in the concentration of linalool. The other odor compounds tested did not evoke an excitatory response from this cell, even at dilutions up to 1:100. Volatiles from a whole tomato leaf and a mineral oil blank also failed to elicit an excitatory response from this PN.
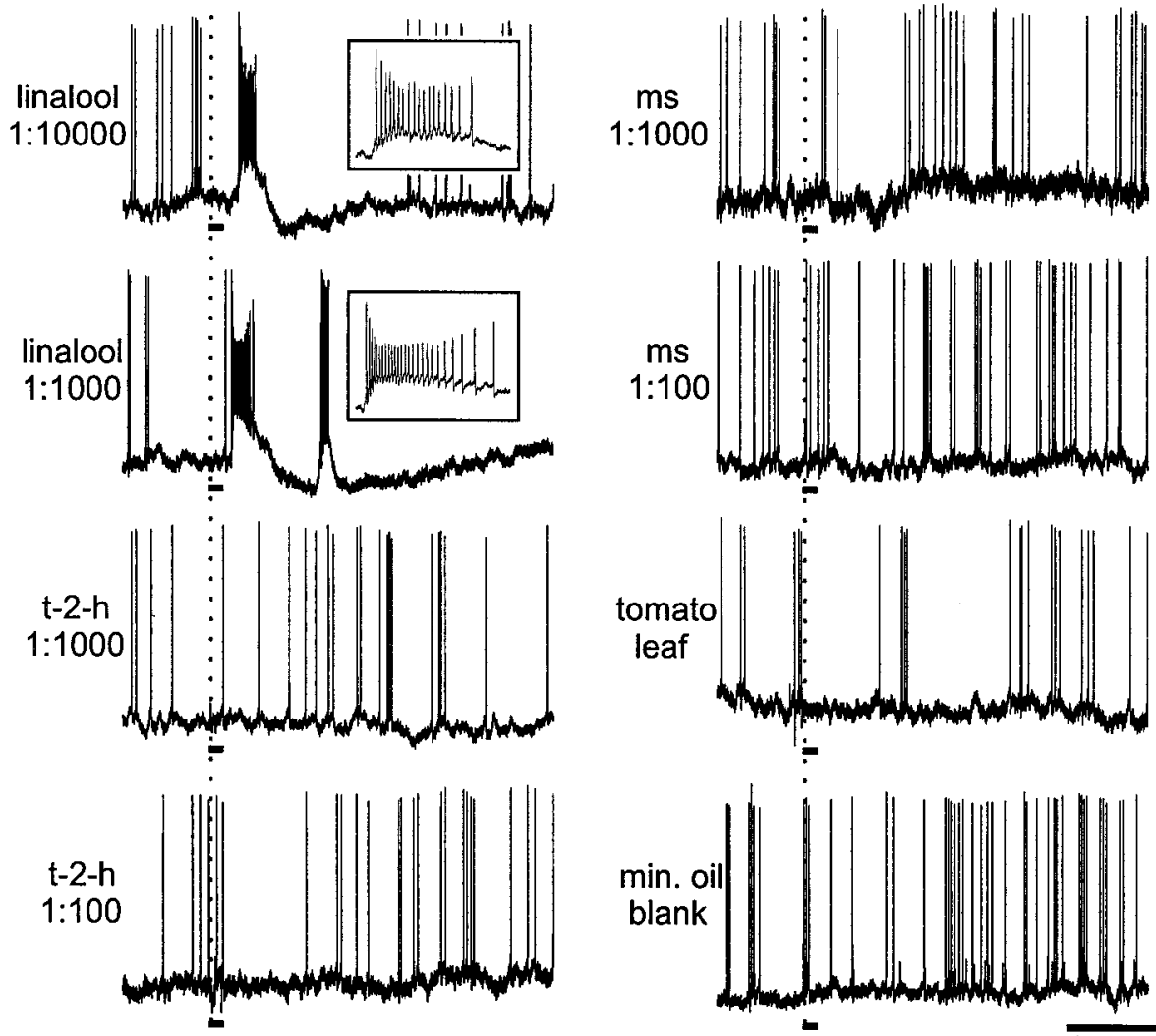

of many plants (Knudsen et al., 1993), including favored host plants of M. sexta (Buttery et al., 1987; Andersen et al., 1988; Loughrin et al., 1990; Raguso and Willis, 1997; Raguso and Pichersky, 1999). Tomato plants are one of the preferred oviposition sites for $M$. sexta (Yamamoto and Fraenkel, 1960). If the LFGs are involved in processing olfactory information related to oviposition, one might expect tomato-leaf volatiles to be odor stimuli for LFG-PNs. In the present study, although linalool evoked consistent, excitatory responses from latLFG-PNs, the headspace volatiles emitted by an isolated tomato leaf did so for only 2 of the 10 latLFG-PNs included in this study (Fig. 7). Among possible explanations for this apparent discrepancy are that either the volatiles emitted by an isolated tomato leaf in our olfactometer probably varied significantly from experiment to experiment or that a single leaf (as used
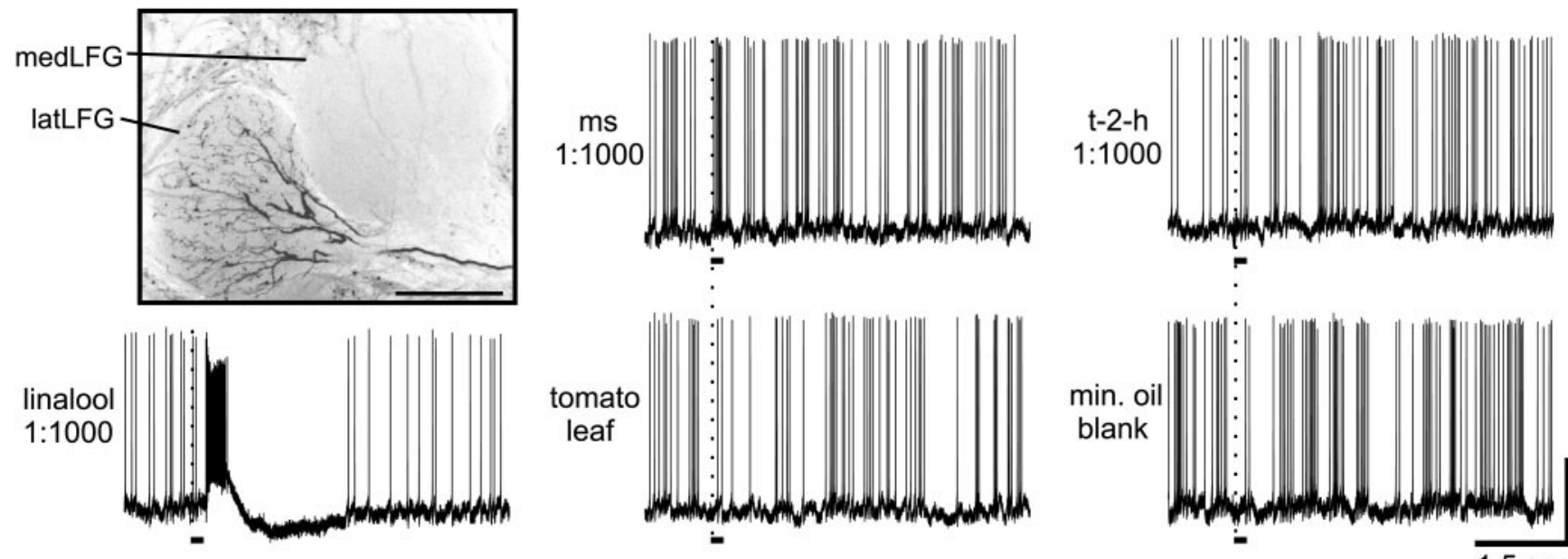

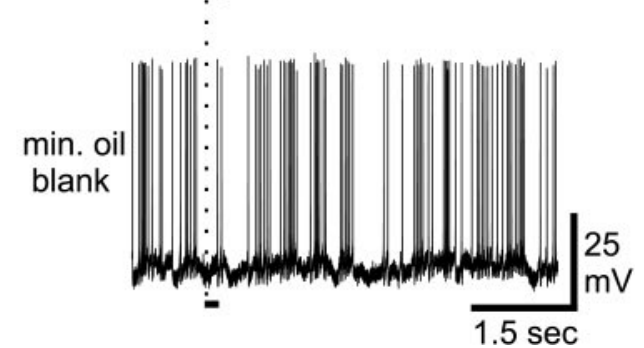

Figure 6. Morphology and physiological properties of another latLFG-PN. The panel in the top left corner is a confocal stack through a section showing the two LFGs. The dendritic arborization of this single, uniglomerular PN was restricted to the latLFG. Scale bar, $50 \mu \mathrm{m}$. Image was inverted in Corel Photo-Paint. Representative physiological records demonstrate that this latLFG-PN was strongly excited by linalool, whereas the other single compounds $(t-2-h$ and $m s)$, as well as the blend of volatiles from a tomato leaf and the mineral oil blank, failed to cause any change in background spiking activity. The response to linalool was biphasic, consisting of an excitatory phase followed by a period of hyperpolarization. The response profile obtained from this latLFG-PN is almost identical to the pattern of responses illustrated in Figure 5. Stimulus onset is indicated by the vertical dotted line. Stimulus duration was $200 \mathrm{msec}$, as indicated by the solid line under each record. 
Table 1. Physiological responses of latLFG-PNs when stimulated with different concentrations of linalool

\begin{tabular}{lllll}
$\begin{array}{l}\text { Dilution of } \\
\text { linalool }\end{array}$ & $\begin{array}{l}\text { Peak ISF } \\
\text { (spikes/sec) }\end{array}$ & Latency $^{b}(\mathrm{msec})$ & $\begin{array}{l}\text { Duration of } \\
\text { excitation }^{c}(\mathrm{msec})\end{array}$ & $\begin{array}{l}\text { Duration of } \\
\text { inhibition }^{d}(\mathrm{msec})\end{array}$ \\
\hline $1: 10,000$ & $87 \pm 22$ & $371 \pm 45$ & $178 \pm 29$ & $1382 \pm 212$ \\
$1: 1000$ & $189 \pm 13$ & $275 \pm 16$ & $359 \pm 23$ & $2745 \pm 384$ \\
$1: 100$ & $215 \pm 19$ & $241 \pm 13$ & $676 \pm 43$ & $4789 \pm 1331$
\end{tabular}

Means \pm SEM from five latLFG-PNs that were tested with all three concentrations of linalool.

${ }^{a}$ Peak instantaneous spike frequency measured during the excitatory phase of the linalool response.

${ }^{b}$ Latency from the beginning of the stimulus pulse to the onset of the excitatory phase of the response.

${ }^{c}$ Duration of the excitatory phase of the linalool response.

${ }^{d}$ Duration of the inhibitory phase of the linalool response, measured from the end of the excitatory response to the return of background firing.

in our experiments) might have released a level of linalool that is below the threshold for excitation of the latLFG-PNs.

Another possible function for the LFGs is that they might be responsible for processing information about components of a male pheromone. Male moths of many species possess scent organs that produce and disseminate male pheromones (Birch et al., 1990). Given the relatedness between the LFGs and the glomeruli of the MGC, as described above, it is possible that the LFGs may be involved in detection of a pheromone released by courting males. Male pheromone is usually produced by sequestering compounds from plants eaten as larvae and therefore is often made up of a mixture of volatiles normally associated with plants (Birch et al., 1990). In fact, linalool is one of the components of the male pheromone produced by a different moth species, Trichoplusia ni (Landolt and Heath, 1990). One of our future goals will be to determine the composition of the emissions from a putative scent organ in the abdomen of male $M$. sexta. In addition, determining the response specificity of PNs with arborizations in the other LFG, the medLFG, may shed light on the functional role of the LFGs and may suggest the suite of odorants that can effect female-specific behaviors.

Reproducible and consistent tuning of individual, identified glomeruli is to be expected if the glomeruli of primary olfactory centers are organized chemotopically. Extending and complementing our previous studies of the glomeruli of the male-specific MGC, our current studies of the latLFG suggest that glomeruli in addition to those of the MGC consistently exhibit limited molecular receptive ranges and may even constitute labeled line repre-

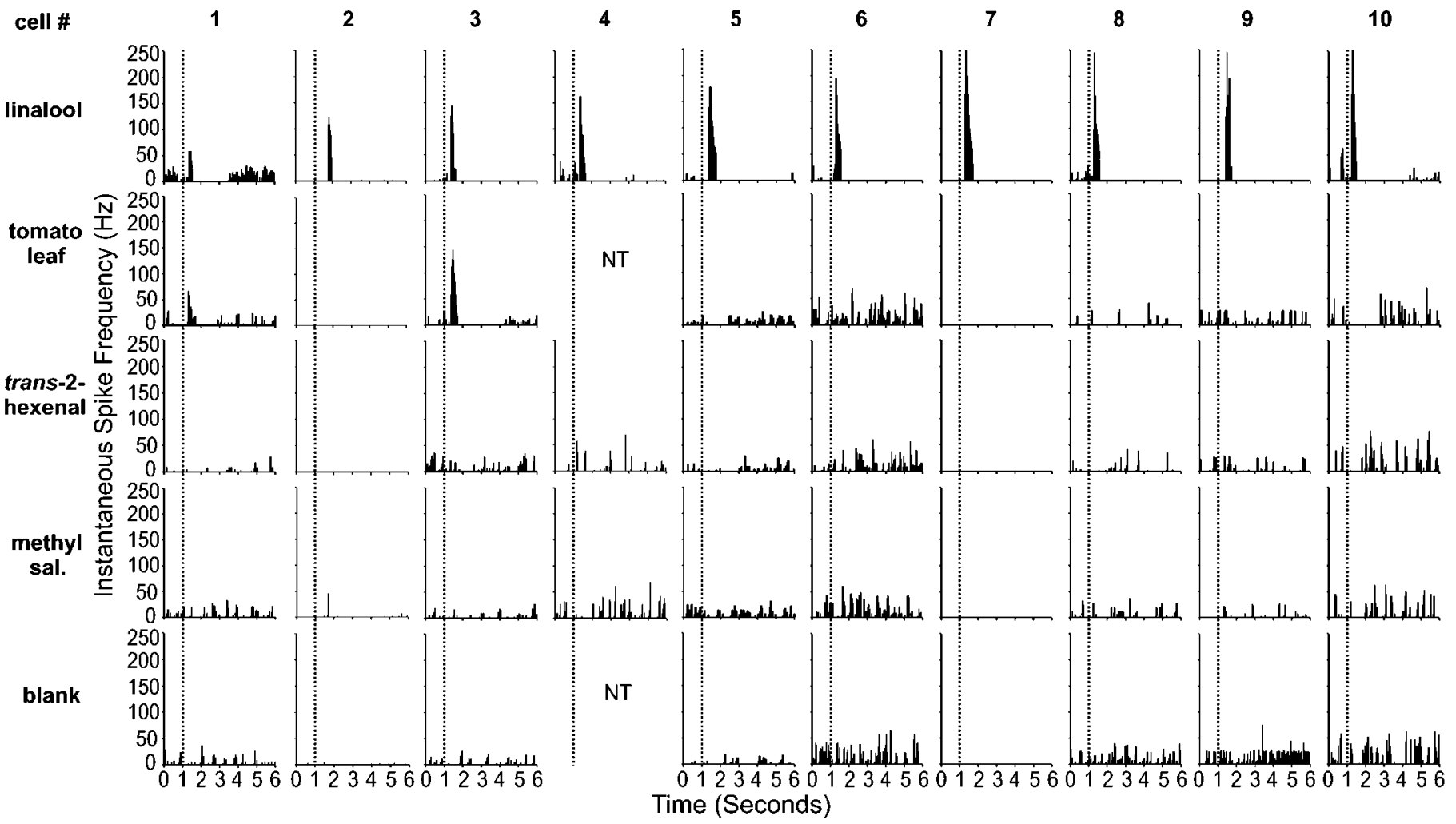

Figure 7. Plots of instantaneous spike frequency versus time for the 10 latLFG-PNs included in this study. Shown are representative records from each neuron when stimulated with linalool, trans-2-hexenal and methyl salicylate, each at a concentration of 1:1000. Also shown are representative responses from each neuron when it was stimulated with the volatile blend from a whole tomato leaf and the mineral oil blank. PNs were numbered in order of ascending peak instantaneous spike frequencies. Stimulus onset is indicated by the vertical dashed line in each column. The stimulus was a single, 200 msec odor pulse. All latLFG-PNs exhibited a marked excitatory response when the ipsilateral antenna was stimulated with linalool, with instantaneous spike frequencies reaching up to $250 \mathrm{sec}^{-1}$ (top row). Two of the 10 latLFG-PNs were also excited by the tomato leaf volatiles (second row, neurons 1 and 3). None of the other compounds tested elicited an excitatory response from these PNs. NT, Not tested. 

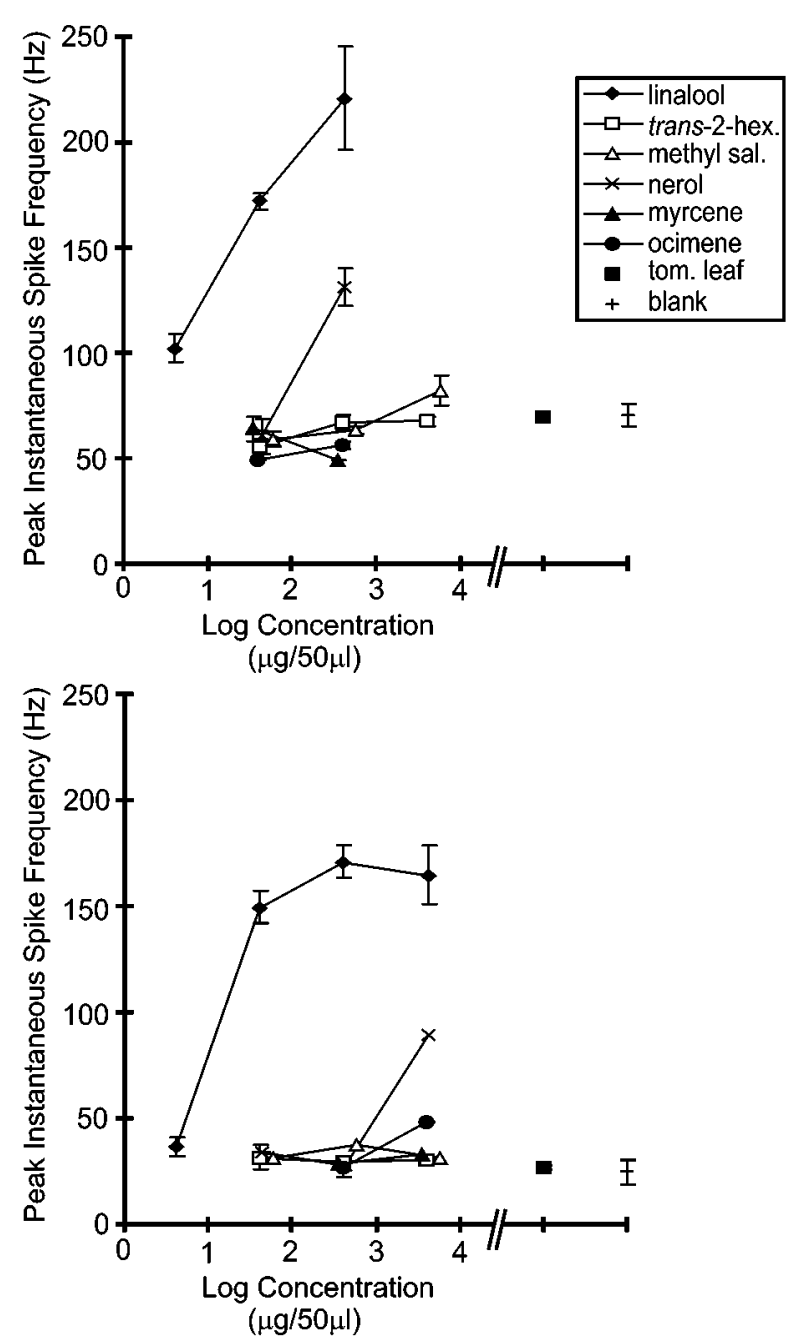

Figure 8. Dose-response curves for two latLFG-PNs. To explore their response characteristics, some of the latLFG-PNs were tested with a wider selection of monoterpenoids (Fig. 1) at various concentrations. Dose-response curves were constructed by plotting the mean peak instantaneous spike frequency (means \pm SEM) versus concentration. The plots show that linalool was the strongest stimulus for both neurons. Nerol could also elicit an excitatory response from these cells, but a much higher concentration was required, and the peak instantaneous frequency was still substantially lower than that attained during the excitatory response to linalool. These two latLFG-PNs differed in their background firing rates and their overall sensitivities to linalool. The PN in the top panel (PN 6 in Fig. 7) exhibited a higher background firing rate $\left(\sim 50 \mathrm{sec}^{-1}\right)$ and greater overall sensitivity to linalool in comparison to the $\mathrm{PN}$ in the bottom panel (PN 5 in Fig. 7).

sentations of particular behaviorally significant odorants. Our findings indicate that within and among individuals, uniglomerular PNs (output neurons) with arborizations in a particular glomerulus exhibit similar response properties when tested with a small but varied panel of odorants. Although the latLFG appears to have a relatively narrow molecular receptive range, reflecting the tuning characteristics of the ORCs that converge on that glomerulus, it remains to be seen whether other glomeruli in the AL have more or less narrow molecular receptive ranges.

In mammals, ORCs that express the same olfactory receptor protein project to a very small number of glomeruli, and it is possible that each glomerulus receives input from ORCs that express a single or closely related receptor type(s) (for review, see
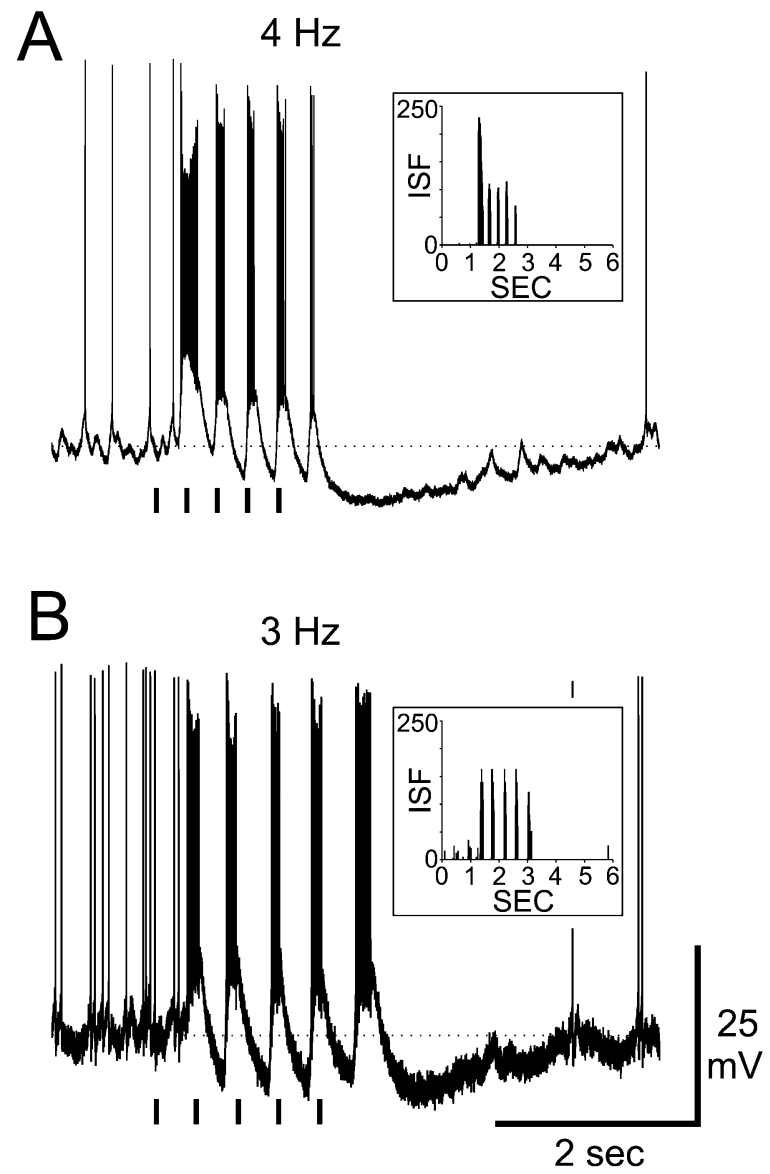

Figure 9. Temporal resolution of linalool pulses by two latLFG-PNs. $A$, Response of a latLFG-PN (PN 10 in Fig. 7) on stimulation of the ipsilateral antenna with linalool (1:1000) delivered at a rate of four pulses per second. $B$, Response of a latLFG-PN (PN 6 in Fig. 7) to linalool (1:1000) delivered at a rate of three pulses per second. Each pulse was 50 msec in duration, as indicated by the bars under each record. Insets in each panel are plots of instantaneous spike frequency $(I S F)$ versus time for the physiological record. In both cases, the latLFG-PN closely followed the presentation of the pulsed stimulus.

Buck, 1996). In moths, ORCs that respond selectively to individual sex pheromone components project exclusively to specific glomeruli of the MGC (Hansson et al., 1992; Christensen et al., 1995; Hildebrand, 1996; Hildebrand and Shepherd, 1997). It will be of interest to determine whether this is also the case for the LFGs and other ordinary glomeruli in the AL of M. sexta. Demonstrating that single glomeruli receive common inputs and yield similar outputs promises to provide a clearer understanding of how olfactory information is represented in the brain and to shed light on the function of the olfactory glomeruli of diverse animals.

\section{REFERENCES}

Andersen RA, Hamilton-Kemp TR, Loughrin JH, Hughes CG, Hildebrand DF, Sutton TG (1988) Green leaf headspace volatiles from Nicotiana tabacum lines of different trichome morphology. J Agric Food Chem 36:295-299.

Birch MC, Poppy GM, Baker TC (1990) Scents and eversible scent structures of male moths. Annu Rev Entomol 35:25-58.

Buck LB (1996) Information coding in the vertebrate olfactory system. Annu Rev Neurosci 19:517-544.

Buonviso N, Chaput MA (1990) Response similarity to odors in olfactory bulb output cells presumed to be connected to the same glomer- 
ulus: electrophysiological study using simultaneous single-unit recordings. J Neurophysiol 63:447-454.

Buttery RG, Ling LC, Light DM (1987) Tomato leaf volatile aroma components. J Agric Food Chem 35:1039-1042.

Chambille I, Masson C, Rospars JP (1980) The deutocerebrum of the cockroach Blaberus craniifer Burm. Spatial organization of the sensory glomeruli. J Neurobiol 11:135-157.

Chaput MA (1990) Is the olfactory bulb functionally organized in parallel columns? In: Chemosensory information processing (Schild D, ed), pp 247-258. Berlin: Springer.

Christensen TA, Hildebrand JG (1987) Male-specific, sex pheromoneselective projection neurons in the antennal lobes of the moth Manduca sexta. J Comp Physiol [A] 160:553-569.

Christensen TA, Hildebrand JG (1988) Frequency coding by central olfactory neurons in the sphinx moth Manduca sexta. Chem Senses 13:463-477.

Christensen TA, Hildebrand JG (1997) Coincident stimulation with pheromone components improves temporal pattern resolution in central olfactory neurons. J Neurophysiol 77:775-781.

Christensen TA, White J (2000) Representation of olfactory information in the brain. In: Neurobiology of taste and smell (Finger TE, Silver WL, Restrepo D, eds). New York: Wiley, in press.

Christensen TA, Harrow ID, Cuzzocrea C, Randolph PW, Hildebrand JG (1995) Distinct projections of two populations of olfactory receptor axons in the antennal lobe of the sphinx moth Manduca sexta. Chem Senses 20:331-323.

Christensen TA, Heinbockel T, Hildebrand JG (1996) Olfactory information processing in the brain: encoding chemical and temporal features of odors. J Neurobiol 30:82-91.

Christensen TA, Waldrop BR, Hildebrand JG (1998) Multitasking in the olfactory system: context-dependent responses to odors reveal dual GABA-regulated coding mechanisms in single olfactory projection neurons. J Neurosci 18:5999-6008.

Distler PG, Bausenwein B, Boeckh J (1998) Localization of odorinduced neuronal activity in the antennal lobes of the blowfly Calliphora vicina: a $\left[{ }^{3} \mathrm{H}\right] 2$-deoxyglucose labeling study. Brain Res 805:263-266.

Friedrich RW, Korsching SI (1997) Combinatorial and chemotopic odorant coding in the zebrafish olfactory bulb visualized by optical imaging. Neuron 18:737-752.

Friedrich RW, Korsching SI (1998) Chemotopic, combinatorial, and noncombinatorial odorant representations in the olfactory bulb revealed using a voltage-sensitive axon tracer. J Neurosci 18:9977-9988.

Hansson BS, Christensen TA (1999) Functional characteristics of the antennal lobe. In: Insect olfaction (Hansson BS, ed), pp 126-161. Berlin: Springer.

Hansson BS, Christensen TA, Hildebrand JG (1991) Functionally distinct subdivisions of the macroglomerular complex in the antennal lobe of the male sphinx moth Manduca sexta. J Comp Neurol 312:264-278.

Hansson BS, Ljungberg H, Hallberg E, Löfstedt C (1992) Functional specialization of olfactory glomeruli in a moth. Science 256:1313-1315.

Heinbockel T, Kaissling K-E (1996) Variability of olfactory receptor neuron responses of female silkmoths (Bombyx mori L.) to benzoic acid and ( \pm )-linalool. J Insect Physiol 42:565-578.

Hildebrand JG (1995) Analysis of chemical signals by nervous systems. Proc Natl Acad Sci USA 92:67-74.

Hildebrand JG (1996) Olfactory control of behavior in moths: central processing of odor information and the functional significance of olfactory glomeruli. J Comp Physiol [A] 178:5-19.

Hildebrand JG, Shepherd GM (1997) Mechanisms of olfactory discrimination: converging evidence for common principles across phyla. Annu Rev Neurosci 20:595-631.

Homberg U, Montague RA, Hildebrand JG (1988) Anatomy of antenno-cerebral pathways in the brain of the sphinx moth Manduca sexta. Cell Tissue Res 254:255-281.

Joerges J, Küttner A, Galizia CG, Menzel R (1997) Representation of odours and odour mixtures visualized in the honeybee brain. Nature 387:285-288.

Kauer JS, Cinelli AR (1993) Are there structural and functional modules in the vertebrate olfactory bulb? Microsc Res Tech 24:157-167.

King JR, Christensen TA, Hildebrand JG (1998) Host-plant odor processing by antennal lobe projection neurons in female Manduca sexta. Chem Senses 23:572.
Knudsen JT, Tollsten L, Bergström LG (1993) Floral scents: a checklist of volatile compounds isolated by head-space techniques. Phytochemistry 33:253-280.

Landolt PJ, Heath RP (1990) Sexual role reversal in mate-finding strategies of the cabbage looper moth. Science 249:1026-1028.

Loughrin JH, Hamilton-Kemp TR, Andersen RA, Hildebrand DF (1990) Headspace compounds from flowers of Nicotiana tabacum and related species. J Agric Food Chem 38:455-460.

Matsumoto SG, Hildebrand JG (1981) Olfactory mechanisms in the moth Manduca sexta: response characteristics and morphology of central neurons in the antennal lobes. Proc R Soc Lond B Biol Sci 213:249-277.

Mori K, Shepherd GM (1994) Emerging principles of molecular signal processing by mitral/tufted cells in the olfactory bulb. Semin Cell Biol 5:65-74.

Raguso RA, Pichersky E (1999) A day in the life of a linalool molecule: chemical communication in a plant-pollinator system. Part 1: linalool biosynthesis in flowering plants. Plant Species Biol 14:95-120.

Raguso RA, Willis MA (1997) Floral scent and its role(s) in hawkmoth attraction. Chem Senses 22:774-775.

Rodrigues V (1988) Spatial coding of olfactory information in the antennal lobe of Drosophila melanogaster. Brain Res 453:299-307.

Rodrigues V, Buchner E (1984) [ $\left.{ }^{3} \mathrm{H}\right] 2$-Deoxyglucose mapping of odorinduced neuronal activity in the antennal lobes of Drosophila melanogaster. Brain Res 324:374-378.

Rospars JP (1983) Invariance and sex-specific variations of the glomerular organization in the antennal lobes of a moth, Mamestra brassicae, and a butterfly, Pieris brassicae. J Comp Neurol 220:80-96.

Rospars JP, Hildebrand JG (1992) Anatomical identification of glomeruli in the antennal lobes of the male sphinx moth Manduca sexta. Cell Tissue Res 270:205-227.

Rospars JP, Hildebrand JG (2000) Sexually dimorphic and isomorphic glomeruli in the antennal lobes of the sphinx moth Manduca sexta. Chem Senses, in press.

Rössler W, Tolbert LP, Hildebrand JG (1998) Early formation of sexually dimorphic glomeruli in the developing olfactory lobe of the brain of the moth Manduca sexta. J Comp Neurol 396:415-428.

Rössler W, Randolph PW, Tolbert LP, Hildebrand JG (1999) Axons of olfactory receptor cells of trans-sexually grafted antennae induce development of sexually dimorphic glomeruli in Manduca sexta. J Neurobiol 38:521-541.

Sanes JR, Hildebrand JG (1976) Structure and development of antennae in a moth, Manduca sexta. Dev Biol 51:282-299.

Schneider D, Wunderer H (1990) Olfactory brain structures of two moth species with dual or male pheromone systems: Creatonotos, Galleria. Proceedings of 10th International Symposium on Olfaction and Taste (Døving KB, ed), pp 159-164. Norway: University of Oslo.

Schneiderman AM, Hildebrand JG, Brennan MM, Tumlinson JH (1986) Trans-sexually grafted antennae alter pheromone-directed behavior in a moth. Nature 323:801-803.

Selchow KI (1998) Processing of plant-associated odors by a subset of projection neurons in the antennal lobe of the female moth, Manduca sexta. PhD Thesis, University of Arizona.

Shields VDC, Hildebrand JG (1998) Responses from the olfactory receptor cells of trichoid sensilla on the antenna of the female sphinx moth, Manduca sexta. Soc Neurosci Abstr 24:2098.

Teicher MH, Stewart WB, Kauer JS, Shepherd GM (1980) Suckling pheromone stimulation of a modified glomerular region in the developing rat olfactory bulb revealed by the 2 -deoxyglucose method. Brain Res 194:530-535.

Vickers NJ, Christensen TA, Hildebrand JG (1998) Combinatorial odor discrimination in the brain: attractive and antagonist odor blends are represented in distinct combinations of uniquely identifiable glomeruli. J Comp Neurol 400:35-56.

Willis MA, Arbas EA (1991) Odor-modulated upwind flight of the sphinx moth, Manduca sexta L. J Comp Physiol [A] 169:427-440.

Yamamoto RT, Fraenkel GS (1960) The specificity of the tobacco hornworm, Protoparce sexta, to solanaceous plants. Ann Entomol Soc Am 53:503-507. 